\title{
BMJ Open How do Korean nursing students build knowledge? A constructivist grounded theory study
}

\author{
Jung Jae Lee, ${ }^{1}$ Charlotte L Clarke, ${ }^{2}$ Maggie N Carson, ${ }^{2}$ Sook Ching Yang ${ }^{3}$
}

To cite: Lee JJ, Clarke CL, Carson MN, et al. How do Korean nursing students build knowledge? A constructivist grounded theory study. BMJ Open 2018;8:e022050. doi:10.1136/ bmjopen-2018-022050

- Prepublication history for this paper is available online. To view these files please visit the journal online (http://dx.doi. org/10.1136/bmjopen-2018022050).

Received 1 February 2018 Revised 8 June 2018 Accepted 22 June 2018

Check for updates

(C) Author(s) (or their employer(s)) 2018. Re-use permitted under CC BY-NC. No commercial re-use. See rights and permissions. Published by BMJ.

${ }^{1}$ School of Nursing, University of Hong Kong, Hong Kong, Hong Kong

${ }^{2}$ School of Health in Social Science, University of Edinburgh, Edinburgh, UK

${ }^{3}$ School of Medicine, Medical Sciences and Nutrition, University of Aberdeen, Aberdeen, UK

Correspondence to Dr Jung Jae Lee; jungjaejaylee@gmail.com

\begin{abstract}
Introduction Nursing is a knowledge-intensive profession. Therefore, to cope with the demands of the nursing role, nursing students need to become competent in managing information to build nursing knowledge. However, nursing students' knowledge building process is poorly understood. This research aimed to explore (1) nursing students' dynamics of how they process nursing information for knowledge building and (2) nursing students' learning context in South Korea for their knowledge building.

Methods A constructivist grounded theory approach was used for this research. Data collection was achieved through four rounds of intensive individual and group interviews with 16 fourth year nursing students in South Korea. The collected data were coded by initial, focused and theoretical coding methods. Constant comparison analysis between data, codes, memos and categories was applied.
\end{abstract}

Results This research identified knowledge building dynamics consisting of three cognitive processes: connecting with information, deciding to accept information and building knowledge. Five motivational factors, including learners' interest, necessity of information, volition to learn, utility of information and the frequency of information that influence the processes were discovered. Moreover, four knowledge stages of memorising, understanding, synthesising and applying and creating emerged.

Conclusions This is the first empirical study on knowledge building dynamics in educational environments for healthcare professionals. The findings of this research provide nursing educators with a practical model that can be used to improve nursing curricula in facilitating students' knowledge building processes. Moreover, a deeper understanding of sociocultural influences on nursing education can assist educators to adapt and generalise the findings to their pedagogical contexts, providing a culturally sensitive and relevant approach to nursing education.

\section{INTRODUCTION}

Nursing is a knowledge-intensive profession that constantly requires the construction, utilisation and development of knowledge to ensure safe and professional care provision. ${ }^{1}$ With the additional recent development of information technology that enabled easy

\section{Strengths and limitations of this study}

- This research identified the nursing students' knowledge building dynamics, including empirical definitions of information and knowledge, based on the epistemological viewpoint of constructivism.

By rigorously implementing constructivist grounded theory methodology, this research developed a theoretical model of knowledge building dynamics of nursing students.

- The sample was final year student nurses, whose knowledge building dynamics may differ from those of more junior students, limiting the relevance of this study to more experienced students only.

- Due to the cultural uniqueness of the setting for this study and the context-bound relevance of qualitative findings, repetition of the study is required in other countries and cultures.

access to and sharing of information, nurses need to manage large amounts of healthcare information for their practice and knowledge building on a daily basis. ${ }^{23}$ Accordingly, in order to qualify as nurses and to handle the current demands of the nursing role, ${ }^{4}$ students should develop competencies in information processing as it plays a vital role in knowledge construction. ${ }^{5}$ However, several reports still reveal that some newly qualified nurses struggle with inadequate nursing knowledge and skills in their new roles due to ineffective knowledge building during their undergraduate education. ${ }^{6}$ Yet, little is known about students' cognitive knowledge building processes within the context of nursing education. ${ }^{8}$ Moreover, there is a lack of definition of what information and knowledge are, and these terms are often used interchangeably and unclearly in healthcare literature. Existing definitions of 'information' and 'knowledge' in nursing are borrowed from other disciplines, such as those from the Data-Information-Knowledge-Wisdom (DIKW) model. ${ }^{9}$ However, only a few attempts have been made to critically review or redefine them to ensure their 
relevancy to nursing studies. Moreover, the DIKW model that is dominant in the nursing field was developed with positivist viewpoints, ${ }^{10}{ }^{11}$ which hindered development of ideas regarding what information and knowledge are through other ontological and epistemological viewpoints (eg, constructivism).

Meanwhile, research paradigms based on traditional cognitive science have been popular with researchers who seek to investigate and understand education for healthcare professionals. ${ }^{12}$ Although cognitive science has achieved success in explaining learning as learners' inner processing of information, it has also received criticism. ${ }^{13}$ Cognitive scientists view the entire learning process exclusively as a mental activity and do not consider the influence of social and cultural contexts on learning, while constructivists believe that learning occurs when learners interact with their environment; thus, they emphasise the importance of context in the construction of individual knowledge. ${ }^{14}$ In addition, recent cognitively based medical education studies highlighted the need to understand 'how' resources involved in learners' mental learning processes work in real contexts, rather than simply 'what' the resources are. ${ }^{12}$ Constructivists' beliefs therefore offer an in-depth understanding and a holistic perspective on nursing students' knowledge building processes by considering the social and cultural contexts in which they learn. Other than the argument of paradigm, the difference in social and cultural contexts between the West and East results in a difference in learning strategies. ${ }^{15} 16$ This is because culture has an undeniable influence on one's identity, which also defines one's learning beliefs. ${ }^{15}$ As such, educational activities that are wholly borrowed from another cultural context can be problematic in both their implementation and maintenance due to the conflict of cultural characteristics, ultimately impeding the effectiveness of students' learning. ${ }^{17} 18$ Therefore, recognition of the social and cultural characteristics that impact on how students build knowledge will be essential for optimum curriculum development so as to maximise nursing students' learning potential.

This research aimed to (1) define the terms of information and knowledge in nursing using empirical data with a constructivist viewpoint and (2) develop a theoretical model that explains the ways in which nursing students process nursing information for knowledge building as a cognitive learning process, and the effect of the South Korean learning context on their knowledge building.

\section{METHODS \\ Design}

Grounded theory allowed us to explore the students' experiences of knowledge building and to develop the theory from the data. ${ }^{19}$ In particular, Charmaz's grounded theory orientated within a constructivist paradigm (ie, constructivist grounded theory: CGT) is a popular methodology in the disciplines of nursing, education and sociology. ${ }^{20}$ This is because CGT provides an in-depth and holistic understanding of experience for theory construction by factoring in sociocultural contexts. ${ }^{20}$ Therefore, a qualitative research strategy guided by CGT was employed as (1) this research aims to construct a theoretical model to explore nursing students' experiences of knowledge building dynamics by considering the South Korean context, (2) the educational paradigm has shifted to constructivism and (3) the researchers' epistemological paradigm is constructivism (ie, ensuring methodological congruence in this research).

\section{Participants}

The inclusion criteria were students who were in their fourth and final year of an undergraduate nursing course (all undergraduate nursing curricula in South Korea run for 4 years and consist largely of two parts: nursing theory courses and clinical placements) and had taken both nursing theory classes and clinical education. However, registered nurses who enrolled in nursing undergraduate courses to earn a bachelor's degree (previous diploma holders) and nursing students who had previous nursing education experience in other countries were excluded. Using purposeful sampling, 20 nursing students from four universities in Seoul, South Korea were contacted via email. Ten nursing undergraduate students from four universities were initially recruited. Subsequently, six nursing students were recruited through theoretical sampling ${ }^{20}$ during data collection (ie, 16 participants in total). The mean age of the participants was 21.88 years $(\mathrm{SD}=1.63)$, ranging from 21 to 27 years, with 14 female and 2 male students.

\section{Data collection}

Four rounds of intensive individual and group interviews (23 interviews in total) conducted in Korean from April 2013 to June 2015 with 16 participants enabled the researcher to adjust the scope of interview topics by focusing on constructing a theoretical framework. ${ }^{20}$ The semistructured interviews incorporated open-ended and non-judgemental questions pertaining to this research topic (eg, 'Tell me about your learning experience in your nursing course', 'How do you usually build nursing knowledge?', 'What does information and knowledge mean to you?' and 'Tell me about your experience in Korean nursing education'). Interviews, each lasting one and half hours, were conducted in meeting rooms in universities or hospitals and were audio-recorded.

The first author, JJL, conducted all interviews. JJL graduated from a nursing undergraduate course and worked as a registered nurse in South Korea for 7 years, during which time he actively engaged in nursing undergraduate education through the delivery of lectures and supervision of clinical placements. Therefore, JJL has an in-depth understanding of educational contexts in South Korea with an insider's viewpoint. JJL also pursued higher degrees in nursing in the UK, enabling JJL to acquire external viewpoints that were useful in understanding the distinctiveness of the Korean educational culture. The collected interview data were interpreted in view of these experiences (ie, reflexivity).$^{20}$ During and after the 
interviews, JJL wrote memos reflecting his ideas and experience regarding the participants' answers. ${ }^{20}$

\section{Ethical considerations}

JJL provided written information about the research and obtained written informed consent from all participants before conducting interviews. No incentives for participation were offered.

\section{Data analysis}

Interview recordings were transcribed and then translated from Korean to English. Language and underlying meanings in the language play a critical role in qualitative research. ${ }^{21}$ In order to preserve the meanings during translation (ie, Korean to English), five bilingual Koreans were asked to provide a systematic translation and back-translation process.

The transcripts were coded using NVivo 11, guided by the three coding stages of CGT (ie, initial, focused and theoretical coding) $\cdot{ }^{20}$ In the initial coding stage, core characteristics of the interview data were extracted by line-by-line and in-vivo coding methods ${ }^{20} 22$ to generate initial codes with gerund forms. Second, focused coding was used to develop core categories from the codes from the initial coding stage.$^{20}$ Last, theoretical coding was used to enable identification of the possible relationships between the categories. Throughout the coding stages, constant comparison analysis between data, codes, memos and categories was applied until data saturation. After generating focused and theoretical codes, the participants reviewed and confirmed the codes through member-checking (ie, sending of interview transcripts and brief analyses to participants for their confirmation) as co-construction of meanings between researchers and participants is crucial in CGT. During the coding stages, the researchers developed a theoretical framework that the participants verified during the fourth round of interviews. The methodological pathway of this study is schematised in figure 1.

\section{Rigour}

Rigour plays a crucial role in qualitative research, ensuring the value of the research results. ${ }^{23}$ This research was conducted systematically according to Charmaz's criteria (ie, credibility, originality, resonance, usefulness). ${ }^{20} \mathrm{~A}$ systematic analytic process, including constant comparison and member-checking, was adopted to minimise translational limitations and enhance the credibility. The originality of the findings in this research was examined by reviewing the existing literature. For resonance, we believe that the students' experiences of knowledge building were explained sufficiently, as the interviews continued until theoretical saturation was reached and the meanings that the students took for granted were identified. The students' general as well as distinguished knowledge-building experiences were included. Thus, the findings have practical value and can be applied to improve nursing education (ie, usefulness).

\section{Patient and public involvement}

Patients were not involved in this study. The study participants were nursing students only. The authors sent the interview transcript and brief analysis to each participant via email so that the participants can member-check the transcript and analysis and have an opportunity to comment on these.

\section{RESULTS}

What information and knowledge mean to nursing students

To understand the participants' knowledge building dynamics, it was imperative to establish what the terms 'information' and 'knowledge' meant to them. The

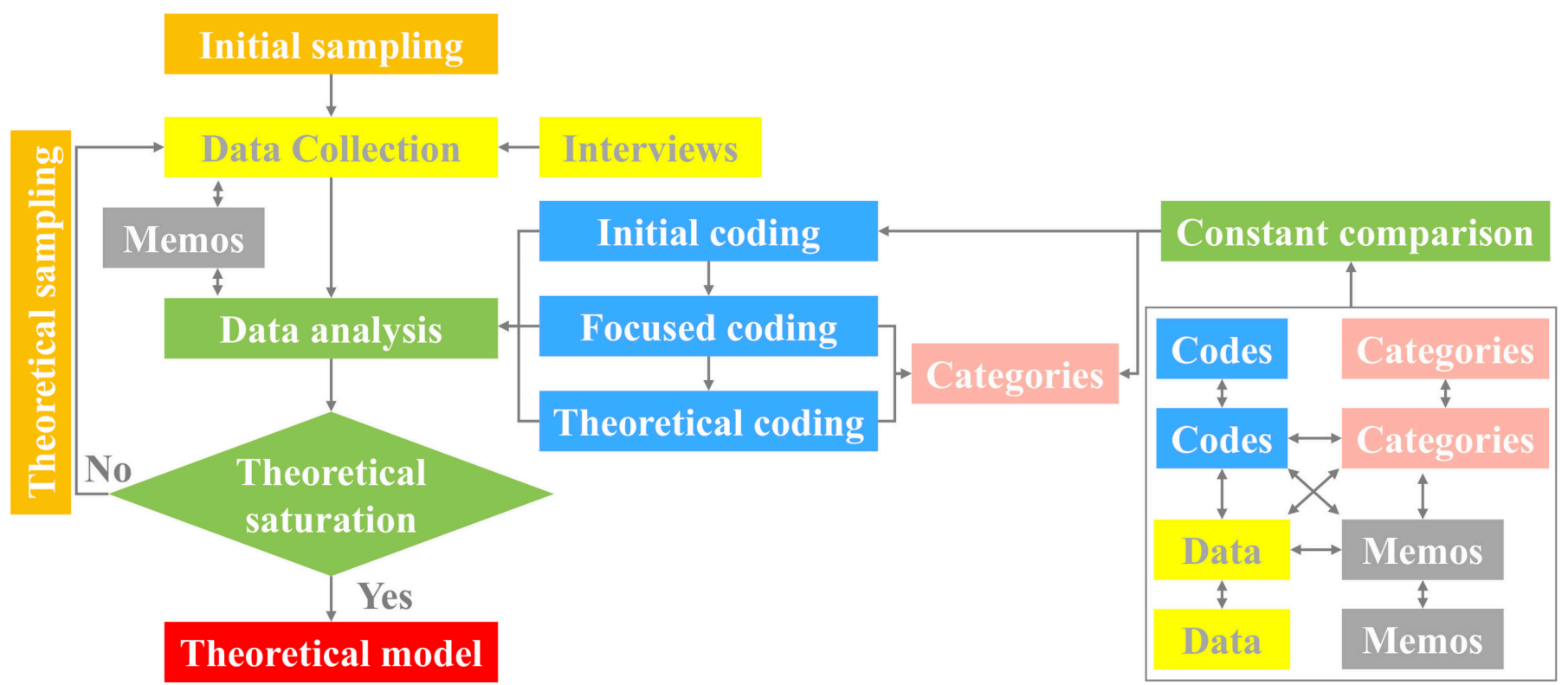

Figure 1 Methodological pathway of this research (ie, data collection and analysis process of this research). This pathway is guided by the constructivist grounded theory approach. 
participants in this study defined the terms 'information' and 'knowledge' clearly and consistently. They distinguished information from knowledge through the concepts of non-subjectivity and subjectivity, respectively. They described information as existing outside and independent of themselves and indicated that they are not engaged with the information (ie, non-subjectivity). Conversely, they described knowledge as existing within themselves (ie, subjectivity).

Information just exists, outside of me... I believe what I know is my knowledge, which cannot be known by others. Therefore, knowledge is a very subjective thing, but information is not. (Participant 3)

Moreover, the participants agreed that knowledge is personal; thus, if another person shares specific knowledge, it is not rendered as knowledge but rather as information. In this way, knowledge can be understood as one's first-hand perspective of information (ie, subjectivity).

When I share my knowledge with others, the knowledge becomes information to the others. This is because others objectively receive my knowledge. (Participant 11)

Information and knowledge can also be understood through the relationship of ownership. When information is in one's possession, it is considered as his/her knowledge, whereas when information is not in one's possession, it remains as information.
I think information is to be obtained [from outside of myself], but knowledge is to be possessed. (Participant 4)

While the differences between information and knowledge are clear, the participants also noted an interrelationship between the two concepts. As 'knowledge is the subjectification of information' (Participant 1), it is a primary source for knowledge building. The relationship between information and knowledge can be understood as an internal cognitive process whereby information becomes personally embedded.

A fact is information. [Information] does not contain my judgement about that fact. If I add my judgement to that fact, it becomes knowledge. (Participant 1)

These definitions and relationships between information and knowledge are shown in figure 2.

\section{Process of knowledge building}

This research identified three phases of the process of knowledge building ('connecting with information', 'deciding to accept information' and 'building knowledge').

Information exists as a source of knowledge; therefore, the knowledge building process starts with connecting with information, which is in one's external world. Participants connected with nursing information through printed textbooks and digital sources.

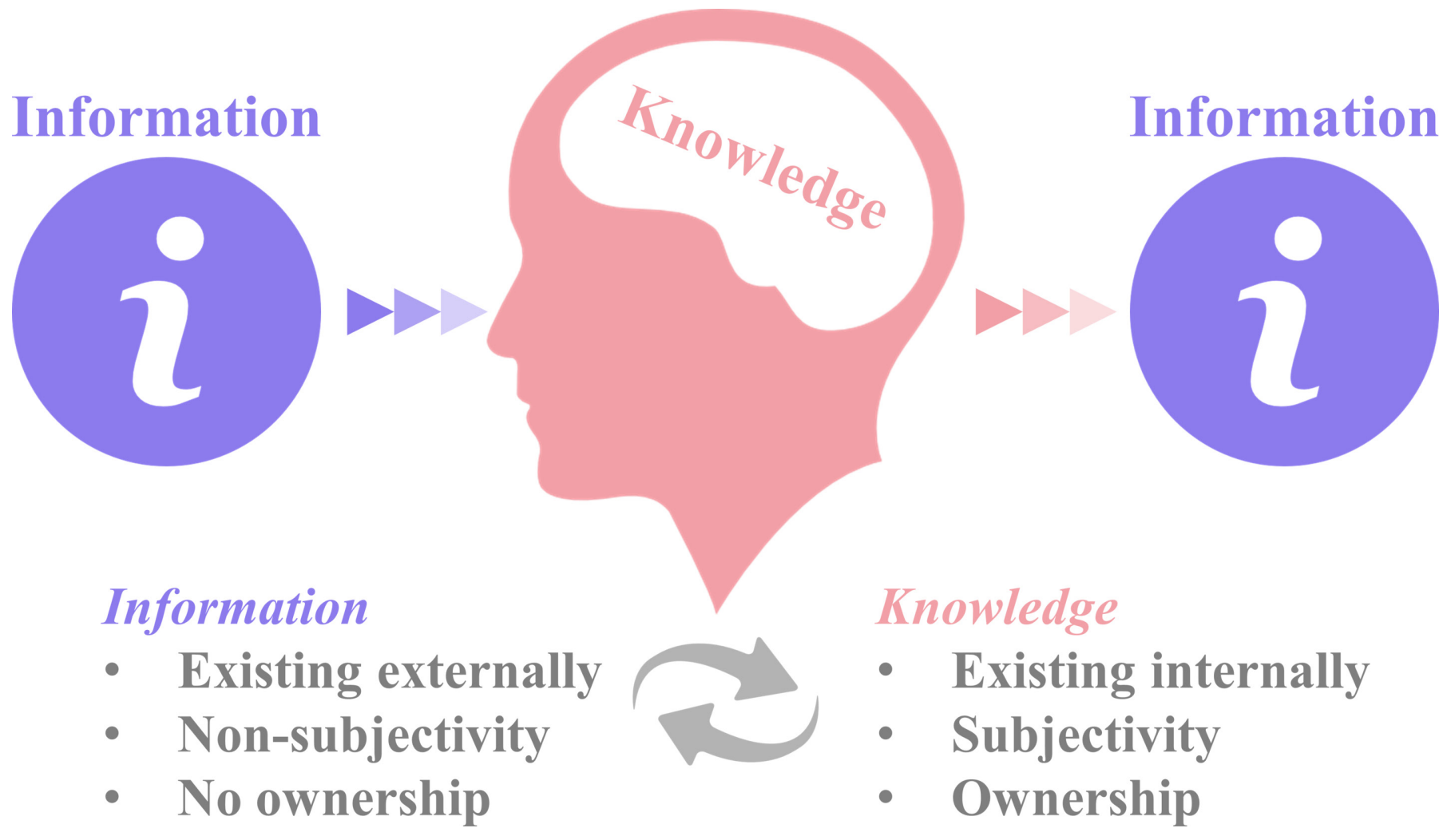

Figure 2 The definitions and relationships between information and knowledge. This figure explains the definitions of information and knowledge and its horizontal and non-hierarchical relationships. The definitions are oriented by the epistemological perspective of constructivism. 


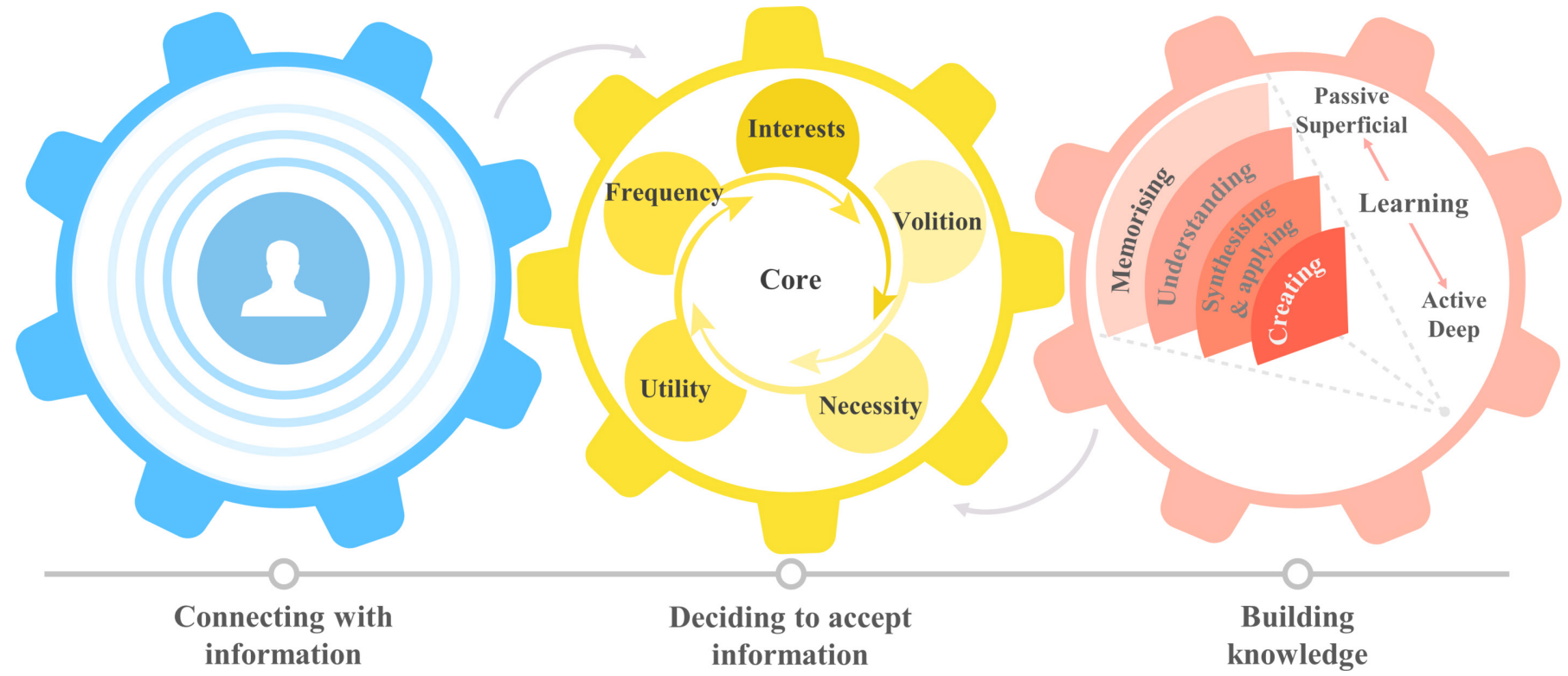

Figure 3 Theoretical model: Cogwheel Knowledge Building Dynamics. The dynamics consist of three cognitive processes: 'connecting with information', 'deciding to accept information' and 'building knowledge'. In the process of deciding to accept information, learners' five motivational factors are explained. In addition, four knowledge stages are demonstrated in the process of building knowledge.

I mainly obtain [nursing] information from textbooks and the internet for my nursing knowledge. (Participant 7)

Due to the plethora of nursing information available through various mediums, it is nearly impossible to connect with every piece of information in a clear and comprehensive way to build knowledge. Thus, the participants undertook an active decision-making process to make decisions about whether to take ownership of the information with which they connect to build knowledge.

Knowledge is taking that [information] and making a decision about it to make it my own. (Participant 1)

\section{Motivational factors}

The decision-making process is motivated by five factors: learners' interest, necessity of information, volition to learn, utility of information and the frequency of information (see figure 3).

\section{Interests in information}

Specific information that was interesting to the participants was more likely to be recalled and actively pursued, and that information would be used to build knowledge.

The more interested I am [in certain information], the more I will think about that particular information, allowing me to go through a feedback process [which also results in the building of knowledge].

(Participant 10)

Therefore, amid a sea of information, interest motivates nursing students to choose information with which they want to connect and which they want to use to build nursing knowledge.
Necessity of information

The necessity of information also influences the decision-making process and contributes to the transformation of information to knowledge. The participants divided information into either necessary or unnecessary.

If I feel [the information] is important or absolutely necessary, I try to remember it. However, if it is just simple or interesting news, it is just temporary [so I don't remember it]. I make knowledge from information that I need. (Participant 2)

As Participant 2 mentioned, deciding on the necessity of the information affected the participants' motivation to actively remember and even understand that information.

\section{Volition to learn}

The participants with strong desires to learn had stronger volitions to learn and therefore were more likely to actively seek out information as well as actively use that information to build knowledge.

First, I think my attitude is very important. If I do something passively, I obtain just a little [information], whereas if I make an effort to learn a lot, I can obtain a lot... (Participant 5)

\section{Utility of information}

When the participants believed that the information with which they connect had a high utility rate, they were more motivated to select and transform that information into knowledge.

If I know I will utilise this information for my presentations or in my practice later with real patients, 
it will eventually become knowledge, as the information will be continuously utilised. However, if not, it will not become knowledge. (Participant 12)

\section{Frequency of connecting with information}

When the participants frequently and repeatedly connected with certain information, the information tended to linger in their minds. Within a particular period of time, the number of connections made with certain information influenced the participants' decisions to build knowledge.

If I don't connect with the information frequently, I will forget it. (Participant 1)

Regardless of the participants' intentions, repeated connections with information reinforced the decision-making process and increased the chances of information becoming knowledge. Although this factor is the least active process, it is nonetheless one of the five factors that influence the transformation of information to knowledge.

During the interviews, the participants did not identify only one factor that influenced their decision-making process; rather, they identified several factors that work together simultaneously. Overall, more correlations and intersections between the factors increase the motivation to build knowledge.

Moreover, the five motivational factors can be divided into intrinsic and extrinsic factors. Interest and volition can be regarded as intrinsic factors, whereas the necessity, utility and frequency can be viewed as extrinsic factors (see table 1). As exemplified previously, intrinsic reasons for learning include the individual student's autonomy

Table 1 Interview quotations of intrinsic and extrinsic factors of motivation

\begin{tabular}{lll}
\hline & Factors & Quotations \\
\hline $\begin{array}{l}\text { Intrinsic } \\
\text { factors }\end{array}$ & Interest & $\begin{array}{l}\text { My individual interests influence } \\
\text { [the knowledge building } \\
\text { process]. (Participant 1) }\end{array}$ \\
& Volition & $\begin{array}{l}\text { The volition to learn is } \\
\text { just the beginning [of my } \\
\text { learning]. (Participant 5) }\end{array}$ \\
Extrinsic & Necessity & $\begin{array}{l}\text { The contexts around me demand [the } \\
\text { acquirement of necessary nursing } \\
\text { information]. (Participant 13) }\end{array}$ \\
& Utility & $\begin{array}{l}\text { I obtained information about } \\
\text { neuroscience care nursing. If I predict } \\
\text { to utilise this information during } \\
\text { my presentations or in my nursing } \\
\text { placements later, it will become } \\
\text { knowledge. (Participant 12) }\end{array}$ \\
& & $\begin{array}{l}\text { If I frequently find the same } \\
\text { information during my clinical } \\
\text { placements, it can be my knowledge. } \\
\text { (Participant 14) }\end{array}$ \\
& &
\end{tabular}

and own satisfaction. In contrast, extrinsic reasons are related more to the contextual demands. The study found that the participants were more likely to endorse extrinsic factors during their nursing education. This is characteristic of people within a collectivist culture, such as Korea.

I definitely didn't decide to study nursing because I wanted to...But my parents wanted... Others also said that while it is hard to get employed in Korea, I would easily get employed if I do nursing. (Participant 9)

\section{Building knowledge: stages of knowledge}

Although the participants made relatively conscious decisions about what information should become knowledge, not all built knowledge was the same. This research identified four different stages of knowledge that exist concurrently. These are derived from four knowledge building methods: memorising, understanding, synthesising and applying and creating (see figure 3).

\section{Memorising}

The participants indicated memorisation as the primary method used to study nursing.

I have to memorise, without question. I sit down, open my textbook, and then make summaries. I usually study like this. (Participant 1 )

Despite acknowledging memorisation as the most common study method in nursing education, the participants agreed that it generates 'the lowest stage of nursing knowledge' (Participant 11). The participants understood that memorised information existed in their minds without any of their own feedback or interpretation; therefore, it was more easily forgotten or disregarded. Memorisation is a passive learning method that is traditional and prevalent in Korean educational culture.

\section{Understanding}

The participants defined understanding as an authentic and beneficial form of knowledge building and agreed that understanding is a key aspect of the knowledge building process that results in a higher stage of knowledge compared with memorisation.

Understanding is surely a superior concept [to that of memorisation]. Memorisation is just like taking a picture, but I can't see what is inside the picture. Understanding means knowing how the picture was taken. (Participant 7)

According to the picture metaphor offered by Participant 7 , the knowledge gained from understanding includes the information that nursing students decide to accept at face value and the related causal and influential information. Thus, understanding, as opposed to memorisation, allows for a more expansive and comprehensive output of knowledge.

In nursing education, particularly education during clinical placements, knowledge via understanding enables 
nursing students to think critically and act appropriately in the unpredictable clinical contexts. Such thinking is not provoked through memorisation. Thus, knowledge built from understanding reflects a basic but essential knowledge that nursing students in clinical environments need to possess.

\section{Synthesising and applying}

Synthesising and applying of knowledge is the third stage of knowledge building, as defined by the study participants.

By synthesising the pre-existing knowledge, or adding new knowledge to old knowledge, the level of knowledge can be promoted. (Participant 11)

If I use the knowledge attained from memorisation on my patients, it is known as application. However, some students cannot apply the knowledge they gained through memorisation during clinical placements. Therefore, I think application [of knowledge] is something different [an ability]. (Participant 7)

Such a process enables flexible thinking based on knowledge attained from memorisation and understanding and requires active applications of such knowledge. For this stage of knowledge to be reached, prior knowledge attained from former stages serves as the background or basic knowledge that is required for this stage. Thus, this stage of knowledge depends on lower stages of knowledge and the previously discussed knowledge building mechanisms.

I think that memorisation or understanding must take precedence for synthesis and application to occur. The synthesis and application process will only be possible if there is memorisation and understanding. (Participant 5)

\section{Creating}

Knowledge creation is the fourth method and the participants identified the knowledge built through this method as the highest stage of knowledge.

If I mix and combine knowledge and then produce new knowledge, it is a higher stage of knowledge. (Participant 7)

Descriptions of knowledge at this stage by the participants are rare. It is assumed that the nursing students were restricted from reaching this stage of knowledge creation due to the emphasis on memorisation stressed in Korean educational contexts.

\section{Knowledge building as a cogwheel process}

The process of knowledge building can be conceptualised as a theoretical framework and model of knowledge building dynamics (figure 3 ). The process does not follow a rigid step-by-step forward process (ie, connecting with information $\rightarrow$ deciding to accept information $\rightarrow$ building knowledge); instead, it runs continuously and simultaneously, like three cogwheels shown in figure 3. For example, when the 'deciding to accept information' cogwheel runs, it influences both the 'connecting with information' cogwheel and the 'building knowledge' cogwheel. Similarly, when the 'building knowledge' cogwheel runs, it also influences the 'connecting with information' cogwheel. New knowledge and previous experience can be connected to form additional information or knowledge and vice versa. This means each stage in the process can activate other phases.

\section{DISCUSSION}

This empirical research aimed to define information and knowledge within the nursing context and to develop a theoretical model to explain nursing students' cognitive learning process for knowledge building within the South Korean context.

The interpretation of the study findings is based on the researchers' epistemological paradigm of constructivism.

In the DIKW model that is popularly adopted in nursing fields, information and knowledge are represented in a hierarchical order, such that the lower order form is a source for a higher order form (eg, information is a source of knowledge).${ }^{10} 11$ While the process of transforming information to knowledge in this research resembled the DIKW model, the information-knowledge relationship based on the epistemological viewpoint of constructivism in this research was found to be horizontal and non-hierarchical. Other than epistemological consideration, Alavi and Leidner ${ }^{24}$ in the discipline of management, similarly reported that a back-and-forward process exists between information and knowledge, confirming the reciprocal dynamics between the two, as illustrated in figure 2. Interestingly, however, most of the literature defined information and knowledge based on philosophical and theoretical assumptions rather than empirical evidence. This research offers evidence to support the philosophical and theoretical assumptions, as the findings of this research are derived from empirical research data.

This research discussed the nursing students' learning process, from connecting with information to the building of knowledge based on their decisions to accept certain information. As an individual's knowledge represents information that has been internalised and subjectified, the individual needs to consciously select the information around him or her to build knowledge. The philosophical concept of 'intentionality' can explain nursing students' cognitive decisions regarding information. Searle ${ }^{25}$ explained intentionality as the subject's mental activity (eg, thought, judgement and awareness) involving the object that exists in the world outside of the subject. Without mental activity regarding the object, the subject is not able to notice the object's existence. In this research, the nursing students' intentionality can be regarded as a mechanism to recognise the 'objects' (ie, information 
in this research). As such, knowledge building is a mental activity that starts with recognising information (the object) pertaining to the nursing students (ie, the subject), corresponding with 'connecting with information' and then referencing to the information to accept it to build knowledge, corresponding with 'deciding to accept information'.

In the decision-making process, this research identified five motivational factors that activate the nursing students' cognitive processes of learning and influence the nursing students' intentionality. ${ }^{26}$ A systematic review and meta-analysis of psychological factors related to university students' learning revealed a domain named 'sources of motivation', which represents a learner's motivational reasons to engage in his/her learning process. ${ }^{27}$ This domain was further divided into two categoriesintrinsic and extrinsic motivations. Intrinsic motivation is self-motivation for the pleasure of learning (eg, learning interests) while extrinsic motivation involves actions to obtain instrumental rewards (eg, rewards by a teacher or parents). As in two previous studies, ${ }^{27} 28$ the motivational factors found in this research were divided into intrinsic and extrinsic factors, and the resultsindicated that extrinsic learning factors influenced nursing students more than initially thought. This tendency can be explained by the characteristics of Asian students having been nurtured amid collectivist cultures in Asian countries. ${ }^{29}$ Many educationalists have been discussing the dichotomised attributes of Eastern and Western learners. ${ }^{29-31}$ Eastern learners who are influenced by the Confucian culture are socially oriented and put greater value on satisfying their families, whereas Western learners are oriented to achieve individual goals. ${ }^{32}$ Extrinsic motivations are often described negatively as they can lead to surface learning while intrinsic motivations are considered ideal conditions of deep learning. ${ }^{2732}$ However, extrinsic motivations should not be associated only with surface learning. Asian learners exposed mostly to extrinsic motivational factors would use their own learning strategies to adapt to their context and engage in effective in-depth learning. ${ }^{29}{ }^{30}$ As knowledge building is a social process, ${ }^{33}$ a learner's motivation to build knowledge is related to his or her sociocultural background, in our case, collectivist sociocultural background. This could explain why extrinsic rather than intrinsic motivational factors affected the Korean nursing students who participated in this research.

After making their decisions on which information to accept, nursing students can build nursing knowledge through different stages: memorising, understanding, synthesising and applying and creating. Although no evidence regarding stages of knowledge is available in the existing healthcare education literature, many debates have been conducted on differentiating surface learning from deep learning in the fields of education and educational psychology. Researchers in both fields have identified the properties of surface learning as (1) intending to reproduce information, (2) memorising without establishing links between prior knowledge, (3) passive learning and (4) dreading failure, whereas the defined deep learning as (1) intending to understand, (2) establishing links between prior knowledge, (3) using evidence and (4) having active attitudes towards learning. ${ }^{34-37}$

Based on the above rationale, learning via memorisation is frequently regarded as surface and passive type of learning while learning via understanding is considered a deep and active learning. This idea is reflected in the findings of this research (ie, knowledge gained from understanding rather than memorisation requires more active approaches). The properties of deep learning, or understanding, share some common characteristics with the third stage of knowledge (ie, synthesising and applying), such as establishing links between prior knowledge and using evidence. However, the participating nursing students in this research divided understanding (ie, deep learning) into two detailed concepts, as they believed 'synthesising and applying' required higher cognitive abilities than solely understanding. The participants believed that the knowledge from synthesising and applying is higher due to the nature of nursing studies. As nursing is a practical field, nursing students need to be equipped with the skills needed to synthesise nursing knowledge in complex clinical environments and then apply this knowledge to nursing care. Therefore, attaining a solid knowledge base through memorisation and understanding and subsequently developing the competencies required to apply that knowledge to nursing practice in clinical contexts-an essential educational objective in nursing educationmakes up the third stage of knowledge. However, the Korean students relied more on memorisation during their learning process at the expense of other higher learning goals, such as understanding, synthesising and applying.

Like the extrinsic motivation that many Asian students have been found to have, the phenomenon of relying excessively on memorisation of information is common among Asian students, and it is expected that this phenomenon would cause poor academic results, especially in Western educational contexts. ${ }^{35}$ However, much evidence $^{153839}$ suggests that memorisation is one of the processes involved in understanding; thus, Asian learners who rely more on memorisation should not be considered to learn ineffectively in comparison to Western learners. Particularly, $\mathrm{Li}^{15}$ argued that many Asian students memorise information to understand the material during their learning process (ie, memorisation for higher stages). Therefore, memorisation should be understood as a continuum rather than just surface learning to achieve higher levels of learning. ${ }^{15}$ However, it should be noted that memorisation is still classified as the lowest stage of knowledge in this research, and students in nursing fields are encouraged to achieve higher stages of knowledge, in particular 'synthesising and applying'. Thus, a clinical educational environment, which fosters the construction of higher stages of knowledge, as opposed to building knowledge from memorisation, would benefit nursing 
students' learning and preparation for their professional careers.

\section{Recommendations for nursing education in East Asian countries}

Specific measures can be implemented to motivate students who respond more to extrinsic factors to actively partake in the cognitive process of choosing information for knowledge building. Nursing educators can therefore use clinical case studies, role playing and simulations to help students envisage the necessity and utility of the information required to build their knowledge. Practical procedural skills can be introduced through training courses and revision sessions to increase the frequency of information, and they can be incorporated into summative examinations to imprint on students the need to have sound knowledge of those skills.

As Asian learners use different learning strategies, such as finding meaning in a sociocultural context to conduct deep learning, ${ }^{30-32} 38$ it will be advisable for educators to facilitate those learning strategies by using collaborative assignments and presentations. Students will be more motivated to gather information and build knowledge to benefit others, including themselves. Educators can also create online-forums where students can pose questions and receive class participation grades for their contributions. To pose meaningful questions, students will have to build knowledge on a topic to be able to formulate a question that can challenge fellow nursing students. Activities such as these will encourage the use of knowledge building methods beyond memorisation, resulting in higher stages of knowledge.

\section{Limitations}

This research identified nursing students' definitions of information and knowledge based on constructivist paradigm and the definitions guided the development of knowledge building dynamics model. To our best knowledge, it is the first attempt to identify these definitions with the constructivist viewpoint. Therefore, further studies are required to confirm the definitions as well as the model for the wider use of the findings.

Qualitative research is inherently context specific, and consequently this study is limited by its relevance to the sample and study characteristics (final year students in South Korea). This limitation is, in part, mitigated by including multiple recruitment sites (four Universities) and by a description of the structure and culture of the nursing courses-these details allow for transferability to other contexts.

Data collection was conducted in Korean and processes of translation and back-translation aimed to protect the nuance of the language, but nonetheless there can be some loss of meaning when data are translated across languages and cultures.

\section{CONCLUSION}

This is the first empirical study on knowledge building dynamics in nursing education fields. This study clarified how students define information and knowledge within the nursing field context and provided empirical evidence supporting the relationship between the two concepts, clarifying the students' interpretation of the knowledge building process. The dynamics of cognitive learning identified in this research indicates the need for nursing educators to continue considering the multiple motivational factors involved in their students' learning within specific sociocultural contexts. Student learning would be enhanced, for example, when educators support students with an Eastern educational background to deepen their learning beyond possessing information, using techniques to assimilate this into personally held knowledge. A deeper understanding of sociocultural influences on nursing education can help nursing educators adapt and generalise the findings to their specific pedagogical contexts, providing a culturally sensitive and relevant approach to nursing education.

Contributors All authors have made a direct and substantial contribution to the work reported in the manuscript in the following three areas: (1) conception and design of the study (JJL, CLC, MNC and SCY), data collection (JJL) or analysis and interpretation of the data (JJL, CLC, MNC and SCY); (2) writing the manuscript (JJL and SCY) or providing critical revision of the manuscript for intellectual content (CLC, MNC and SCY) and (3) statistical expertise (N/A); obtaining funding (N/A); administrative, technical or material support (N/A) or supervision (CLC and MNC). All authors have given final approval of the submitted manuscript and agree to allow the corresponding author to serve as primary correspondent with the editorial office.

Funding The authors have not declared a specific grant for this research from any funding agency in the public, commercial or not-for-profit sectors.

Competing interests None declared.

Patient consent Not required.

Ethics approval The research ethics committee in the School of Health and Social Science at the University of Edinburgh approved this research.

Provenance and peer review Not commissioned; externally peer reviewed. Data sharing statement No additional data available.

Open access This is an open access article distributed in accordance with the Creative Commons Attribution Non Commercial (CC BY-NC 4.0) license, which permits others to distribute, remix, adapt, build upon this work non-commercially, and license their derivative works on different terms, provided the original work is properly cited, appropriate credit is given, any changes made indicated, and the use is non-commercial. See: http://creativecommons.org/licenses/by-nc/4.0/.

\section{REFERENCES}

1. McBride AB. The growth and development of nurse leaders. New York: Springer Pub Co, 2011.

2. While A, Dewsbury G. Nursing and information and communication technology (ICT): a discussion of trends and future directions. Int J Nurs Stud 2011;48:1302-10.

3. Intas G, Kostagiolas P, Zavras D, et al. Information-seeking behavior of Greek nursing students: a questionnaire study. CIN: Computers, Informatics. Nursing 2017;35:109-14.

4. Still M. Addressing Library Anxiety (LA) in student nurses: a study in an NHS Foundation Trust Hospital library and information service. Health Info Libr J 2015;32:322-5.

5. McGonigle D, Mastrian K. Nursing informatics and the foundation of knowledge. 3rd ed. Sudbury: Jones \& Bartlett Learning, 2015.

6. Moscaritolo LM. Interventional strategies to decrease nursing student anxiety in the clinical learning environment. J Nurs Educ 2009;48:17-23.

7. Lapkin S, Fernandez R, Levett-Jones T, et al. The effectiveness of using human patient simulation manikins in the teaching of clinical reasoning skills to undergraduate nursing students: a systematic review. JBI Libr Syst Rev 2010;8:661-94. 
8. Anderson JA, Willson P. Knowledge management: organizing nursing care knowledge. Crit Care Nurs Q 2009;32:1-9.

9. Nelson R. Major theories supporting health care informatics. In: Englebardt S, Nelson R, eds. Health Care Informatics: An Interdisciplinary Approach. Maryland, Missouri: Mosby, 2002:3-27.

10. Ronquillo C, Currie LM, Rodney P. The evolution of data-informationknowledge-wisdom in nursing informatics. ANS Adv Nurs Sci 2016;39:E1-E18.

11. Matney SA, Maddox LJ, Staggers N, Susan AM, Lory JM, Nancy S. Nurses as knowledge workers: is there evidence of knowledge in patient handoffs? West J Nurs Res 2014;36:171-90.

12. Mylopoulos M, Regehr G. Cognitive metaphors of expertise and knowledge: prospects and limitations for medical education. Med Educ 2007;41:1159-65.

13. Thagard P. Cognitive science. In: Zalta EN, ed. The Stanford Encyclopedia of Philosophy Fall 2014 Edition. CA: Stanford University, 2014.

14. Anderson T, Dron J. Three generations of distance education pedagogy. The International Review of Research in Open and Distributed Learning 2011;12:80-97.

15. Li J. Cultural foundations of learning: East and West. New York: Cambridge University Press, 2012.

16. Lee $\mathrm{H}-\mathrm{J}$, Lee J, Makara KA, et al. A cross-cultural comparison of college students' learning strategies for academic achievement between South Korea and the USA. Studies in Higher Education 2017;42:169-83.

17. S-n L, D-m F. How culture matters in educational borrowing? Chinese teachers' dilemmas in a global era. Cogent Education 2015;2:1046410.

18. Gervedink Nijhuis CJ, Pieters JM, Voogt JM. Influence of culture on curriculum development in Ghana: an undervalued factor? Journal of Curriculum Studies 2013;45:225-50.

19. Glaser BG, Strauss AL. The discovery of grounded theory: Strategies for qualitative research. London: Aldine Transaction, 1967.

20. Charmaz K. Constructing grounded theory: a practical guide through qualitative analysis. 2nd ed. London: SAGE Publications, 2014.

21. van Nes F, Abma $\mathrm{T}$, Jonsson $\mathrm{H}$, et al. Language differences in qualitative research: is meaning lost in translation? Eur $J$ Ageing 2010;7:313-6.

22. Saldaña J. The coding manual for qualitative researchers. London: Sage, 2015.

23. Creswell JW. Qualitative inquiry \& research design: Choosing among five approaches. 3rd ed. London: SAGE Publications, 2012.

24. Alavi M, Leidner DE. Review: knowledge management and knowledge management systems: conceptual foundations and research issues. MIS Quarterly 2001;25:107-36.

25. Searle JR. Intentionality: an essay in the philosophy of mind. Cambridge Cambridge University Press 1983.
26. Casim DC, Yang Y-C. The development of a conceptual model promoting learners' ownership in an online learning environment. Asian Soc Sci 2012;9:9-14.

27. Richardson M, Abraham C, Bond R. Psychological correlates of university students' academic performance: a systematic review and meta-analysis. Psychol Bull 2012;138:353-87.

28. Pintrich PR, Smith DAF, Garcia T, et al; $A$ manual for the use of the Motivated Strategies for Learning Questionnaire (MSLQ). Ann Arbor, MI: The University of Michigan, 1991.

29. Kember D. Misconceptions about the learning approaches, motivation and study practices of Asian students. High Educ 2000;40:99-121.

30. Biggs JB, Watkins DA. The Chinese Learner in Retrospect. In: Watkins DA, Biggs JB, eds. The Chinese Learner: Cultural, Psychological, and Contextual Influences. Hong Kong, China: Comparative Education Research Centre, 1996:269-85.

31. A-b Y. Ultimate life concerns, self, and Chinese achievement motivation. In: Bond $\mathrm{MH}$, ed. The handbook of Chinese psychology. Hong Kong, China: Oxford University Press, 1996:227-56.

32. Chan CK, Rao N. Revisiting the Chinese learner: Changing contexts, changing education. Hong Kong, China: Springer \& Comparative Education Research Centre, 2010.

33. Mylopoulos M, Scardamalia M. Doctors' perspectives on their innovations in daily practice: implications for knowledge building in health care. Med Educ 2008;42:975-81.

34. Ford N, Miller D, Moss N. Web search strategies and approaches to studying. Journal of the American Society for Information Science and Technology 2003;54:473-89.

35. Entwistle N, Entwistle D. Preparing for examinations: the interplay of memorising and understanding, and the development of knowledge objects. Higher Education Research \& Development 2003;22:19-41.

36. Dinsmore DL, Alexander PA. A critical discussion of deep and surface processing: what it means, how it is measured, the role of context, and model specification. Educ Psychol Rev 2012;24:499-567.

37. Coutinho SA, Neuman G. A model of metacognition, achievement goal orientation, learning style and self-efficacy. Learn Environ Res 2008;11:131-51.

38. Marton F, Dall'Alba GA, Tse LK. Memorizing and understanding: the keys to the paradox? In: Watkins DA, Biggs JB, eds. The Chinese learner: cultural, psychological, and contextual influences. Hong Kong, China: Comparative Education Research Centre, 1996:69-84.

39. Dahlin B, Watkins $D$. The role of repetition in the processes of memorising and understanding: a comparison of the views of German and Chinese secondary school students in Hong Kong. $\mathrm{Br} J$ Educ Psychol 2000;70(Pt 1):65-84. 